\title{
Urteilsfähigkeit von Patienten besser einschätzen
}

Schweizerischer Nationalfonds zur Förderung der wissenschaftlichen Forschung $(S N F)$
Für Ärzte ist es oft schwierig, zu bestimmen, ob ein dementer oder depressiver Patient urteilsfähig ist oder nicht. Das zeigt eine Studie im Rahmen des Nationalen Forschungsprogramms «Lebensende» (NFP 67 unter der Leitung von Prof. Dr. Nikola BillerAndorno). Nun will die Zentrale Ethikkommission der Schweizerischen Akademie der Medizinischen Wissenschaften neue Beurteilungsgrundsätze ausarbeiten.

Die Frage nach der Urteilsfähigkeit von Patienten ist bei jeder medizinischen Therapie entscheidend. Denn nur wer in der Lage ist, die Informationen des Arztes zu verstehen, abzuwägen und zu gewichten, kann eine gültige Einwilligung zu einer Behandlung geben. Ist ein Patient nicht urteilsfähig, muss sich der Arzt gemäss der aktuellen Gesetzgebung auf eine Patientenverfügung abstützen können oder sich an eine vertretungsberechtigte Person wenden.

\section{Dass es bereits verschiedene Leitfäden gibt, um die Urteilsfähigkeit zu bestimmen, ist nur wenigen Ärzten bekannt.}

Korrespondenz:

Dr. phil. med. pract. Manuel Trachsel

Institut für Biomedizinische Ethik und Medizingeschichte Universität Zürich Pestalozzistrasse 24 CH-8032 Zürich Tel. 0786854454 manuel.trachsel[at]uzh.ch
Eine Urteilsunfähigkeit entwickelt sich oft bei Erkrankungen wie Demenz, schweren Depressionen oder nach Hirnverletzungen. Gerade am Lebensende sind viele Menschen aufgrund schwerer Erkrankungen nicht mehr urteilsfähig. Gleichzeitig stehen dann oft komplexe, sehr wichtige medizinische Entscheidungen an: Sollen zum Beispiel lebensverlängernde Massnahmen abgebrochen werden? Oder will ein Patient gar Sterbehilfe in Anspruch nehmen?

\section{Alles andere als trivial}

Die Erhebung der Urteilsfähigkeit ist alles andere als trivial. Der Entscheid hängt etwa von der Situation ab: Eine Person kann zum Beispiel als urteilsfähig gelten, wenn es um alltägliche Entschlüsse wie Kleider oder Essen geht, aber als urteilsunfähig, wenn es um eine schwierige medizinische Entscheidung geht. Zudem können die kognitiven Fähigkeiten, zum Beispiel bei Patienten, die an Alzheimer oder Parkinson erkrankt sind, von Tag zu Tag erheblich schwanken. Zusammen mit seinen Kolleginnen Helena Hermann und Nikola Biller-Andorno hat sich
Manuel Trachsel, Oberassistent am Insitut für Biomedizinische Ethik der Universität Zürich, in einer Fachzeitschrift kürzlich Gedanken gemacht, wie Ärzte die Urteilsfähigkeit unter diesen erschwerten Umständen trotzdem einschätzen könnten [1].

Doch was verstehen Ärzte überhaupt unter Urteilsfähigkeit? Im Rahmen des Nationalen Forschungsprogramms «Lebensende» (NFP 67) haben Trachsel und seine Kolleginnen rund 760 Ärztinnen und Ärzte aus der ganzen Schweiz befragt. Laut den nun veröffentlichten Ergebnissen [2] fühlen sich die allermeisten Ärzte zwar verantwortlich, die Urteilsfähigkeit von Patienten einzuschätzen. Doch selbst von jenen, die sich «sehr verantwortlich» fühlen, denkt nur etwa jeder Dritte, dass er dazu auch genügend kompetent ist.

\section{Faustregeln statt Leitfäden}

Entsprechend uneinig ist sich die Ärzteschaft bei der Definition der Urteilsfähigkeit, bei der Frage, was die wichtigsten Beurteilungskriterien sind, und in der Art, wie sie die Urteilsfähigkeit einschätzen. Die meisten Ärzte haben ihre eigenen Faustregeln, um zu bestimmen, ob ein Patient urteilsfähig ist oder nicht. Dass es bereits verschiedene Leitfäden gibt, um die Urteilsfähigkeit zu bestimmen, ist den wenigsten bekannt.

Die überwiegende Mehrheit der befragten Ärzte gibt aber an, dass sie gerne solche Evaluationsinstrumente nutzen würden. Eine grosse Mehrheit befürwortet zudem klare Richtlinien und bekundet Interesse an Schulungen auf dem Gebiet. Das soll nun geschehen: Basierend auf den Ergebnissen der Umfrage hat die Zentrale Ethikkommission der Schweizerischen Akademie der Medizinischen Wissenschaften (SAMW) entschieden, sich ab nächstem Jahr näher mit dem Thema zu befassen und Grundsätze zur Beurteilung der Urteilsfähigkeit auszuarbeiten.

\section{Literatur}

1 Trachsel M, Hermann H, Biller-Andorno N. Cognitive Fluctuations as a Challenge for the Assessment of Decision-Making Capacity in Patients With Dementia. American Journal of Alzheimer's Disease and other Dementias. 2014; doi: 10.1177/1533317514539377

2 Hermann H, Trachsel M, Mitchell C, Biller-Andorno N. Medical decision-making capacity: knowledge, attitudes, and assessment practices of physicians in Switzerland. Swiss Med Wkly. 2014;144:w14039. 